\title{
The feasibility, acceptability and benefit of interventions that target eating speed in the clinical treatment of children and adolescents with overweight or obesity: A Protocol for a Systematic Review
}

Jennifer S Cox ( $\nabla$ jennifer.cox@bristol.ac.uk)

University of Bristol https://orcid.org/0000-0003-2364-7563

Rebecca Elsworth

NIHR Bristol Biomedical Research Centre Nutrition Theme

Rachel Perry

NIHR Bristol Biomedical Research Centre Nutrition Theme

Julian P. Hamilton-Shield

NIHR Bristol Biomedical Research Centre Nutrition Theme

Elanor C. Hinton

NIHR Bristol Biomedical Research Centre Nutrition Theme

\section{Protocol}

Keywords: Eating rate, speed of eating, intervention, clinic, paediatric, obesity, overweight, treatment

Posted Date: July 20th, 2020

DOI: https://doi.org/10.21203/rs.3.rs-41461/v1

License: (c) (1) This work is licensed under a Creative Commons Attribution 4.0 International License. Read Full License 


\section{Abstract}

Background: Eating speed is positively correlated with higher BMI. Thus, modifying pace of eating may provide a treatment opportunity for those with overweight and obesity, which may have additional, longitudinal benefits if established in childhood. Researchers have designed interventions to help both adults and children reduce eating speed as a mechanism for weight-loss. These interventions include those that change the food that is eaten, those that use technology to support a slower eating speed, and those that work to change eating speed by cognitive means. This review seeks to understand the feasibility, acceptability and benefit to using eating speed in paediatric populations who have experienced such methods as part of a clinical weight-management programme.

Methods: Clinical studies that use eating speed interventions as a treatment for paediatric patients (age 5-18 years) with overweight or obesity will be included in this review. No limits to language or search date will be implemented. A systematic search of the literature will be conducted using the following databases - MEDLINE, PyschINFO and EMBASE via OVID, Web of Science and JBI, Database of systematic reviews and Implementation reports, along with trial registers NICE, ClinicalTrials.gov and Cochrane Central Register of Controlled Trials. A grey literature search will be conducted via OpenGrey, and by writing to experts in the field. Study quality will be assessed and meta-analyses for trials that use similar interventions and report similar outcomes will be undertaken where possible.

Discussion: This review will synthesise evidence regarding the feasibility, acceptability and benefit of using eating speed interventions to develop a narrative review. An assessment of heterogeneity will be assessed and where there is sufficient data, we will carry out a meta-analysis; for example, on the secondary outcomes of BMISDS or eating speed change following the interventions.

\section{Background}

Childhood overweight and obesity is prevalent in increasing numbers of young people (1). Obesity has a complex and multifaceted aetiology, including genetic, behavioural and environmental contributions, with evidence showing a connection between eating at a faster speed and overweight and obesity (26). Studies in school children $(7,8)$ and clinical paediatric patients $(2,6)$ found that those who were heavier were more likely to report eating quickly; a finding corroborated through a systematic review (3). Differences are also seen amongst those who are most overweight, with young females with severe obesity scoring lower on the slowness in eating subscale on the Child Eating Behaviour Questionnaire (CEBQ) than their peers with obesity (6).

Satiety signals are generated initially through gastro-intestinal distension and subsequently through production of satiety hormones within the gut $(9,10)$. By eating quickly, it is thought that more energy can be consumed before satiety signals trigger feelings of fullness $(4,11)$. The same food consumed over a longer duration was associated with higher concentrations of post-prandial anorexigenic gut peptides PYY and GLP-1 (12) and supressed ghrelin (13) when compared with the same food consumed over a short duration. These changes in hormonal concentrations were correlated with higher self-reported feelings of fullness (12) and greater blood-oxygen level dependent (BOLD) activation of both reward and satiety regions of the brain (13). Therefore, eating quickly may limit the ability to eat in line with sensations of satiety. Indeed, paediatric patients with obesity with low slowness in eating scores, also show poor satiety responsiveness (6).

Slower eating may also support improved memory of recent eating episodes, resulting in greater self-reported satiety following an eating episode (14) and in some $(13,15)$, but not all studies (14), reduced subsequent consumption. Once recent study found a $25 \%$ decrease in snack intake following a slowly consumed meal compared to the same meal eaten at a faster rate. This decrease in intake was accompanied by a better recall of the consumed portion size (13).

Eating speed is thought to be maintained with relative consistency across meals (16) and is considered to be a heritable behavioural trait (17). Indeed, those who eat quickly in young childhood have a greater risk of overweight at age six years (18). Slowing of eating speed may therefore provide a treatment opportunity $(5,6)$ and may have additional, longitudinal benefits if established in childhood (17). Researchers have designed interventions to help both adults and children reduce eating speed as a mechanism for weight-loss $(5,19-21)$. These interventions broadly sit within three categories: (i) interventions that change the food that is eaten, (ii) those that use technology to support a slower eating speed, and (iii) those that work to change eating speed by cognitive means alone.

1. Interventions that manipulate the texture of foods have focused on slowing down eating rate by changing foods for harder-to-chew alternatives (22) and also reformulating foods to create new meals that are more difficult to eat quickly (i.e. thickening porridge) (22).

2. Technological devices have been designed that support, and in some instances 'train', the user to slow down their eating rate. Tableware such as forks with integrated sensors that detect movement rate have been developed $(23,24)$, as have weighing scales, such as the Mandolean ${ }^{\circledR}$, that detect the speed in which food is removed from the plate, and prompt the user to pay attention to feelings of satiety $(25,26)$.

3. Interventions that target eating speed through cognition include focused, attentive or mindful eating interventions that draw attention to the sensory qualities and to savouring the food, in the process slowing eating speed (27).

Whilst these interventions have all elicited slower eating speed in controlled laboratory studies, less is known about their applicability as clinical treatments, and more specifically, little is known about their clinical application for paediatric populations. Previous work has raised concerns about the acceptability and feasibility of engaging paediatric clinical populations with such interventions (28).

\section{Aims}

The question addressed by this review is built around the PICO (Patient, Intervention, Comparator, Outcome) framework (29). This systematic review will synthesise evidence regarding the acceptability and feasibility (outcome) of eating speed interventions (intervention) in paediatric populations who have 
experienced such methods as part of a clinical weight-management programme (participant). Secondly, this review will present details of any clinical benefits these interventions have, such as a reduction of intake and subsequent weight-loss, and thirdly, any unwanted side effects created by their use. Together, this data will form a picture of the current status of eating speed interventions as used in paediatric weight management. Should these interventions be deemed to be feasible and acceptable, they may provide a beneficial addition to clinical, weight-maintained programmes that often do not currently target eating behaviours in a structured way.

\section{Methods}

This review is registered on the Prospective Register of Systematic Reviews (PROSPERO) database (ID no. 192719) in concurrence with the 2015 Preferred Reporting Items for Systematic Reviews and Meta-Analysis Protocols (PRISMA-P). The checklist is attached as Appendix A. The final report will be written in concurrence with the PRISMA 2009 checklist (30).

Published trials that use interventions to reduce eating speed in a clinical setting will be included. Where possible, unpublished data and grey literature including research reports, conference proceedings, PhD theses, preprints, white papers and government reports, will be used to identify further trials for inclusion in the review.

Eligibility criteria

The eligibility criteria for this review is built around the PICO (Patient, Intervention, Comparator, Outcome) framework (29). This review will include both published and unpublished research. No date restrictions will be placed on the search criteria and this review will accept studies in any language, using an external transcription service if required.

Participants

This review will include research in children and adolescents aged between 5 and 18 years, who are receiving clinical treatment for overweight or obesity. Recruitment may have occurred in either primary or secondary care treatment settings. Adult and non-human studies will be excluded.

Intervention

Interventions that target eating speed by any modality namely interventions that manipulate the texture of the food, those that instruct a slower eating speed using technology such as pacing devices (i.e. the Mandolean ${ }^{\circledR}$ or pacing forks) or through cognitive training and skill development interventions, such as mindful eating.

Research involving general mindfulness or other cognitive or therapeutic training that is not specifically targeting eating behaviour will be excluded.

Comparator

As this review predominantly is interested in feasibility and acceptability outcomes, trials with no control group and non-randomised trials will be included alongside randomised controlled trials (RCTs). For studies with a control group, the following comparators would be acceptable: no intervention, waitlist controls or an alternate intervention. There will be no expectation of blinding, due to the difficulty in blinding an eating speed intervention.

Outcome

The primary outcomes for the review will be the feasibility and acceptability of eating-rate interventions, as measured by recruitment (\% of target sample recruited), adherence (\% of intervention followed by participant), fidelity (\% of intervention followed by staff) and completion rates (\% drop-out rate of starting sample). Any reasons provided for the above outcomes will be included and synthesised qualitatively.

Secondary outcomes will be indicators that identify impacts of the intervention, including measures of eating speed (changes to meal duration or pace of eating in minutes/bites-per-minute or as recorded on the Child Eating Behaviour Questionnaire), the amount of food consumed (kcals or grams) and measures of body size (Body Mass Index (BMI), Body Mass Index Standard deviation score) BMISDS/z-score, \% body fat or waist-circumference). Un-wanted side effects or difficulties that arise due to the usage of the intervention will also be extracted; for example, a reduction of fruit and vegetable consumption or an increase in stigma due to using the device.

The data extraction form will be adapted as data is extracted, to ensure all relevant data is captured.

Study design

All types of study design will be accepted, including non-randomised trials, randomised controlled trials (RCTs), observational studies, pre-post studies, case control studies, case-studies, experimental and quasi-experimental studies.

Search strategy

A systematic three-phased search of the literature will be conducted. First, a search will be conducted using the following databases - MEDLINE, PyschINFO and EMBASE via OVID, followed by an analysis of the outputs; titles, abstracts and index terms. This will be checked in collaboration with a librarian to inform the full search. The second phase will involve running the finalised search within the following databases: MEDLINE, PyschINFO, EMBASE, Web of Science and JBI Database of systematic reviews and Implementation reports, along with trial registers NICE, ClinicalTrials.gov and Cochrane Central Register of 
Controlled Trials. A grey literature search will be conducted via OpenGrey, and by writing to experts in the field. The third search phase will involve checking the reference lists of included papers and other relevant systematic reviews for additional references. All authors will be responsible for suggesting additional relevant articles.

The search strategy can be found in Appendix B.

Data management

All data will be managed using Rayyan systematic review software (https://rayyan.qcri.org/welcome)

Selection and extraction

All abstracts will be screened by two independent reviewers. Of these, relevant articles will be retrieved, and two reviewers will be responsible for the full-paper reviews. Reasons for exclusion will be documented. Inconsistencies will be resolved through discussion with a third reviewer.

Data will be extracted using the data extraction form (Appendix C) by two independent reviewers. Data regarding the characteristics of the sample will be extracted (country, sample size, age, demographics and setting), the intervention (type of intervention, duration), comparator details, primary outcomes (recruitment, fidelity, drop-out rate and reasons provided both qualitatively by the patient or as explained by the author) and secondary outcomes (changes to eating speed, energy intake, body size and any unwanted side-effects/problems). Data required to assess methodological quality will also be included where available. Inconsistencies will be deliberated, with decisions including a third reviewer where needed.

Quality checking

The Cochrane Risk of Bias Version 3 tool (Higgins et al., 2011) will be used for randomised controlled trials. The Newcastle-Ottawa quality assessment scale will be used to assess the quality of non-randomised controlled trials (31). Two reviewers will independently assess the quality of each included study.

Data analysis

The data collated from all studies will be synthesised as a narrative review with a summary of extracted data. An assessment of heterogeneity will be conducted and where there is enough data, we will carry out meta-analysis considering continuous variables as weighted mean differences and including $95 \%$ confidence intervals (Cls). Pooled risk ratios (RRs) and $95 \%$ Cls will be calculated for dichotomous outcomes. Analyses will comprise only between-group comparisons. We will use a random effect model for the meta-analysis of results, as we anticipate a high level of heterogeneity among included studies. Degree of statistical heterogeneity will be assessed using the $\mathrm{I}^{2}$ statistic (32). If feasible, sub-group analysis may be conducted, grouping interventions from the three classifications of intervention: food texture, technological, or cognitive.

Dealing with missing data

If the data required are not available in the publication, we will first attempt to back-calculate from the data presented (e.g. numerator or denominator from percentages, standard deviation from standard errors or $95 \% \mathrm{Cls}$ ). If this is not possible, we will attempt to contact the study author

Sensitivity analyses

We will conduct sensitivity analyses to include only the RCTs classified as low risk of bias according to the Cochrane risk of bias tool.

Confidence in cumulative evidence

The strength of the body of research will be assessed using the Grading of Recommendations, Assessment, Development and Evaluation (GRADE) approach (33).

\section{Abbreviations}

BMI - Body Mass Index

BMISDS - Body Mass Index Standard deviation score

BOLD - Blood-oxygen level dependent

CEBQ - Child Eating Behaviour Questionnaire

GLP-1 - Glucagon-like peptide 1

GRADE - Grading of Recommendations, Assessment, Development and Evaluation

GW4- Great West Four

MRC - Medical Research Council

NICE - National Institute for Health and Care Excellence

NIHR - National Institute for Health Research

PICO - Patient, Intervention, Comparator, Outcome

PYY - Peptide YY

PRISMA-P - Preferred Reporting Items for Systematic Reviews and Meta-Analysis Protocols

PROSPERO - Prospective Register of Systematic Reviews

RCTs -Randomised controlled trials 


\section{Declarations}

\section{Ethical approval}

Not applicable

\section{Consent for publication}

Not applicable

\section{Availability of data and materials}

Not applicable

\section{Competing interests}

The authors declare that they have no competing interests.

\section{Funding}

This review is funded by a GW4 MRC Doctoral training programme grant awarded to JC. This research is supported by the NIHR Biomedical Research Centre at University Hospitals Bristol NHS Foundation Trust and the University of Bristol. The views expressed in this publication are those of the authors and not necessarily those of the NHS, the National Institute for Health Research or the Department of Health and Social Care.

\section{Acknowledgements}

Not applicable

\section{Author's information}

Not included

\section{Authors' contributions}

$\mathrm{JC}$ contributed at all stages of the design and writing of this protocol. EH and RE contributed during the design of the systematic review and searches. JHS contributed clinical knowledge to the design of the review and searches. RP contributed expertise in systematic review methodology in the design of the review and searches. All authors read, contributed to and approved the final manuscript.

\section{References}

1. National Child Measurement Programme. Child obesity and excess weight: small area level data - GOV.UK [Internet]. London; 2017 [cited 2018 Sep 3]. Available from: https://www.gov.uk/government/statistics/child-obesity-and-excess-weight-small-area-level-data.

2. Slyper AH, Kopfer K, Huang WM, Re'Em Y. Increased hunger and speed of eating in obese children and adolescents. J Pediatr Endocrinol Metab. 2014 May 1;27(5-6):413-7.

3. http://doi.wiley.com/10.1111/j.1467-789X.2011.00936.x

Mesas AE, Muñoz-Pareja M, López-García E, Rodríguez-Artalejo F. Selected eating behaviours and excess body weight: a systematic review. Obes Rev [Internet]. 2012 Feb 1 [cited 2018 May 15];13(2):106-35. Available from: http://doi.wiley.com/10.1111/j.1467-789X.2011.00936.X.

4. Ohkuma T, Hirakawa Y, Nakamura U, Kiyohara Y, Kitazono T, Ninomiya T. Association between eating rate and obesity: A systematic review and metaanalysis. Vol. 39, International Journal of Obesity. Nature Publishing Group; 2015. p. 1589-96.

5. Robinson E, Almiron-Roig E, Rutters F, de Graaf C, Forde CG, Tudur Smith C, et al. A systematic review and meta-analysis examining the effect of eating rate on energy intake and hunger. Am J Clin Nutr [Internet]. 2014 Jul 1 [cited 2020 May 11];100(1):123-51. Available from:

https://academic.oup.com/ajcn/article/100/1/123/4576445.

6. http://doi.wiley.com/10.1111/cob.12127

Gross AC, Fox CK, Rudser KD, Foy AMH, Kelly AS. Eating behaviours are different in youth with obesity and severe obesity. Clin Obes [Internet]. 2016 Feb [cited 2020 May 11];6(1):68-72. Available from: http://doi.wiley.com/10.1111/cob.12127.

7. Yamagishi K, Sairenchi T, Sawada N, Sunou K, Sata M, Murai U, et al. Impact of speed-eating habit on subsequent body mass index and blood pressure among schoolchildren: The ibaraki children's cohort study (IBACHIL). Circ J. 2018;82(2):419-22. 
8. Lin M, Pan L, Tang L, Jiang J, Wang Y, Jin R. Association of eating speed and energy intake of main meals with overweight in Chinese pre-school children. Public Health Nutr. 2014;17(9):2029-36.

9. 10.1111/j.1467-3010.2009.01753.x

Benelam B. Satiation, satiety and their effects on eating behaviour. Nutr Bull [Internet]. 2009 Jun 1 [cited 2020 May 13];34(2):126-73. Available from: http://doi.wiley.com/10.1111/j.1467-3010.2009.01753.x.

10. Wynne K, Stanley S, McGowan B, Bloom SR. Appetite control. J Endocrinol. 2005;184(2):291-318.

11. Morton GJ, Cummings DE, Baskin DG, Barsh GS, Schwartz MW. Central nervous system control of food intake and body weight. Vol. 443, Nature. Nature Publishing Group; 2006. p. 289-95.

12. $10.1210 /$ jc. $2009-1018$

Kokkinos A, le Roux CW, Alexiadou K, Tentolouris N, Vincent RP, Kyriaki D, et al. Eating Slowly Increases the Postprandial Response of the Anorexigenic Gut Hormones, Peptide YY and Glucagon-Like Peptide-1. J Clin Endocrinol Metab [Internet]. 2010 Jan 1 [cited 2020 May 7];95(1):333-7. Available from: https://academic.oup.com/jcem/article-lookup/doi/10.1210/jc.2009-1018.

13. Hawton K, Ferriday D, Rogers P, Toner P, Brooks J, Holly J, et al. Slow down: Behavioural and physiological effects of reducing eating rate. Nutrients. 2019;11(1):1-23.

14. Ferriday D, Bosworth ML, Lai S, Godinot N, Martin N, Martin AA, et al. Effects of eating rate on satiety: A role for episodic memory? Physiol Behav. 2015 Dec 1;152(Pt B):389-96.

15. Robinson E, Kersbergen I, Higgs S. Eating "attentively" reduces later energy consumption in overweight and obese females. Br J Nutr. 2014 Aug;28(4):657-61. 112(.

16. McCrickerd K, Forde C. Consistency of Eating Rate, Oral Processing Behaviours and Energy Intake across Meals. Nutrients [Internet]. 2017 Aug 17 [cited 2020 May 13];9(8):891. Available from: http://www.mdpi.com/2072-6643/9/8/891.

17. Llewellyn $\mathrm{CH}$, van Jaarsveld $\mathrm{CH}$, Boniface D, Carnell S, Wardle J. Eating rate is a heritable phenotype related to weight in children. Am J Clin Nutr [Internet] 2008 Dec 1 [cited 2020 May 13];88(6):1560-6. Available from: https://academic.oup.com/ajcn/article/88/6/1560/4617115.

18. Agras WS, Kraemer HC, Berkowitz RI, Hammer LD. Influence of early feeding style on adiposity at 6 years of age. J Pediatr. 1990 May;116(5)(1):805-9.

19. Sander H, Roel H. Take It Slow: can feedback from a smart fork reduce eating speed? Front Public Heal. 2016;4.

20. Kadomura A, Tsukada K, Siio I. EducaTableware: Computer-Augmented Tableware to Enhance the Eating Experiences. In: Conference on Human Factors in Computing Systems - Proceedings [Internet]. New York, New York, USA: Association for Computing Machinery; 2013 [cited 2020 May 7]. p. $3071-4$. Available from: http://dl.acm.org/citation.cfm?doid=2468356.2479613.

21. Ford AL, Bergh C, Södersten P, Sabin MA, Foundation R, Hollinghurst S, et al. Treatment of childhood obesity by retraining eating behaviour: randomised controlled trial. BMJ [Internet]. 2010 [cited 2018 Aug 7]; Available from:

22. Forde CG, van Kuijk N, Thaler T, de Graaf C, Martin N. Texture and savoury taste influences on food intake in a realistic hot lunch time meal. Appetite [Internet]. 2013 Jan 1 [cited 2020 May 12];60(1):180-6. Available from: http://www.ncbi.nlm.nih.gov/pubmed/23085683.

23. Kadomura A, Nakamori R, Tsukada K, Siio I. EaTheremin. In: SIGGRAPH Asia 2011 Emerging Technologies, SA'11 [Internet]. New York, New York, USA: ACM Press; 2011 [cited 2020 May 7]. p. 1-1. Available from: http://dl.acm.org/citation.cfm?doid=2073370.2073376.

24. Kadomura A, Li C-Y, Chen Y-C, Tsukada K, Siio I, Chu H. Sensing fork. In: CHI '13 Extended Abstracts on Human Factors in Computing Systems on - CHI EA '13 [Internet]. New York, New York, USA: ACM Press; 2013 [cited 2017 Oct 10]. p. 1551. Available from: http://dl.acm.org/citation.cfm? doid=2468356.2468634.

25. Ford AL, Bergh C, Södersten P, Sabin MA, Hollinghurst S, Hunt LP, et al. Treatment of childhood obesity by retraining eating behaviour: randomised controlled trial. BMJ [Internet]. 2009 Jan 5 [cited 2017 Oct 10];340:b5388. Available from: http://www.ncbi.nlm.nih.gov/pubmed/20051465.

26. Hinton EC, Birch LA, Barton J, Holly JMP, Biernacka KM, Leary SD, et al. Using neuroimaging to investigate the impact of Mandolean ${ }^{\circledR}$ training in young people with obesity: A pilot randomised controlled trial. BMC Pediatr. 2018 Nov 22;18(1).

27. Daly P, Pace T, Berg J, Menon U, Szalacha LA. A mindful eating intervention: A theory-guided randomized anti-obesity feasibility study with adolescent Latino females. Complement Ther Med. 2016 Oct;1:28:22-8.

28. Hamilton-Shield J, Goodred J, Powell L, Thorn J, Banks J, Hollinghurst S, et al. Changing eating behaviours to treat childhood obesity in the community using Mandolean: the Community Mandolean randomised controlled trial (ComMando) - a pilot study. Health Technol Assess (Rockv). 2014;18.

29. BMC Health Services Research 10.1186/s12913-014-0579-0

Methley AM, Campbell S, Chew-Graham C, McNally R, Cheraghi-Sohi S, PICO, PICOS. and SPIDER: A comparison study of specificity and sensitivity in three search tools for qualitative systematic reviews [Internet]. Vol. 14, BMC Health Services Research. BioMed Central Ltd.; 2014 [cited 2020 May 12]. p. 579. Available from: https://bmchealthservres.biomedcentral.com/articles/10.1186/s12913-014-0579-0.

30. Moher D, Liberati A, Tetzlaff J, Altman DG. Preferred reporting items for systematic reviews and meta-analyses: The PRISMA statement. Vol. 339, BMJ (Online). British Medical Journal Publishing Group; 2009. p. 332-6.

31. Wells GA, Shea B, O'Connell D, Peterson J, Welch VLM, et al. Newcastle-Ottawa Scale for assessing the quality of nonrandomized studies in Metaanalysis. 2019 [cited 2020 May 15]; Available from: https://www.researchgate.net/publication/261773681_The_NewcastleOttawa_Scale_NOS_for_Assessing_the_Quality_of_Non-Randomized_Studies_in_Meta-Analysis.

32. Higgins J, Thomas J, Chandler J, Cumpston M, Li T, Page M, Welch V, editors. Cochrane Handbook for Systematic Reviews of Interventions [Internet]. Cochrane Handbook for Systematic Reviews of Interventions. 2019 [cited 2020 Jun 17]. Available from: https://books.google.co.uk/books? 
$\mathrm{hl}=$ en \&lr=\&id=bTqyDwAAQBAJ\&oi=fnd\&pg=PT15\&dq=cochrane+handbook+for+systematic+reviews+of+interventions+version+5.1.0\&ots=hbSBn5rOpf\&s handbook for systematic reviews of int.

33. Guyatt GH, Oxman AD, Vist GE, Kunz R, Falck-Ytter Y, Alonso-Coello P, et al. GRADE: An emerging consensus on rating quality of evidence and strength of recommendations. BMJ [Internet]. 2008 Apr 26 [cited 2020 Jun 26];336(7650):924-6. Available from: https://www.bmj.com/content/336/7650/924.

\section{Supplementary Files}

This is a list of supplementary files associated with this preprint. Click to download.

- Appendices.docx

- PRISMAPchecklistEatingRateSR.docx 